\title{
A Modified EMD-ACWA Denoising Scheme using a Noise-only Model
}

\author{
Ihssene Tellala \\ LIS Laboratory \\ Department of Electronics \\ Ferhat Abbas Setif University 1 \\ Setif, Algeria \\ ihcene-electro@hotmail.fr
}

\author{
Nourredine Amardjia \\ LIS Laboratory \\ Department of Electronics \\ Ferhat Abbas Setif University 1 \\ Setif, Algeria \\ amardjianour@univ-setif.dz
}

\author{
Abderrezzak Kesmia \\ LCCNC Laboratory \\ Department of Electronics \\ Ferhat Abbas Setif University 1 \\ Setif, Algeria \\ arz.kesmia@gmail.com
}

\begin{abstract}
This paper describes a modified denoising approach combining Empirical Mode Decomposition (EMD) and Adaptive Center-Weighted Average (ACWA) filter. The Intrinsic Mode Functions (IMFs), resulting from the EMD decomposition of a noisy signal, are filtered by the ACWA filter, according to the noise level estimated in each IMF via a noise-only model. The noise levels of IMFs are estimated by the characteristics of fractional Gaussian noise through EMD. It is found that this model provides a better estimation of noise compared to the absolute median deviation of the signal used in the conventional method. The proposed EMD-ACWA scheme is tested on simulation and real data with different white Gaussian noise levels and the results are compared with those obtained by the conventional EMD-ACWA, EMD Interval Thresholding (EMDIT) and wavelet methods. Test results show that the proposed approach has a superior performance over the other methods considered for comparison.
\end{abstract}

Keywords-empirical mode decomposition; adaptive center weighted average; noise-only model; signal denoising

\section{INTRODUCTION}

Signal denoising is a key process in speech, image, and other signal processing applications. It enables noise removal to its maximum extent in order to restore the original signal [13]. Linear methods, such as Wiener filtering [4] are among the simplest and commonly used methods to suppress noise from a signal, yet they remain not effective for nonlinear and nonstationary signals which are usually the case of real signals. Noise reduction methods based on wavelets [5-8] have been the dominant techniques for several years. In wavelet domain, the signal enhancement is based on applying a thresholding to wavelet coefficients of the noisy signal. Wavelet based methods are relatively more suitable for non-stationary signal analysis. However, the main drawback of these techniques is that the basic functions are predetermined, which results in mismatching with the varying nature of signals. Empirical Mode Decomposition (EMD) [9] has been extensively used as an alternative approach to analyze non-stationary and nonlinear signals. Unlike wavelet methods, the basic functions of EMD methods are derived directly from the signal itself. The EMD principle is to decompose adaptively a given signal into a finite number of components, called Intrinsic Mode Functions (IMFs) through an iterative process known as "sifting".

In [10-13], the statistical characteristics of white fractional Gaussian noise have been extensively studied by EMD. It was proved that the EMD acts as a dyadic filter bank when applied to various types of noise and therefore is a powerful tool for removing noise from observed data [14-16]. Among the most important methods of EMD-based denoising, is the denoising by reconstructing the signal with previously thresholded IMFs $[16,17]$. In [18], a denoising approach, called EMD interval thresholding (EMD-IT), was developed by applying the wavelet thresholding principle to the IMFs resulting from the EMD decomposition. The threshold was obtained by using the statistical characteristics of the white Gaussian noise through EMD. Authors in $[1,19]$ proposed a novel EMD-based denoising method by applying the adaptive center weighted average (ACWA) filter to the IMFs and provided the term EMD-ACWA. However, their study is limited to only speech signals. The ACWA is a simple filter which has been used largely in white noise suppression in image enhancement applications $[20,21]$. Stationarity of the signal is not necessary for the implementation of ACWA filter because it operates in the time domain.

In the current study, a modified EMD-ACWA method for signal denoising is proposed by adding an improvement to the strategy developed in [1]. The improvement is achieved by estimating the noise contained in each IMF using a noise-only model [11], thus enhancing the accuracy of noise estimation. The proposed EMD-ACWA denoising scheme is tested on real speech signals of CORPORA database [22], real ECG records from the MIT-BIH Arrhythmia database [23], and different simulation signals.

\section{EMPIRICAL MODE DECOMPOSITION}

The EMD method was developed from the assumption that any non-stationary and nonlinear time series consists of different simple IMFs. Each IMF has a distinct time scale and acts as a basis function which must meet the following two conditions: 
- In the whole signal, the number of local extrema must be equal with the number of zero crossings or differ at most by one.

- The average value of the local maxima envelope and the local minima envelope is zero.

The sifting algorithm to obtain IMFs of a signal $x(t)$ could be described as [9]:

1. Find the local extrema (maxima and minima) of the signal $x(t)$.

2. Construct the upper and lower envelopes $E_{\max }(t)$ and $E_{\text {min }}(t)$, respectively, using cubic spline interpolation.

3. Determine the local mean as: $m_{1}(t)=\left[E_{\max }(t)+E_{\min }(t)\right] / 2$

4. Subtract $m_{1}(t)$ from the original signal to form the details $h_{1}(t)=x(t)-m_{1}(\mathrm{t})$.

5. Decide whether $h_{1}(t)$ is an IMF or not by checking the two basic conditions mentioned above.

6. Regarding $h_{1}(t)$ as the new data and repeating steps 1 to 5 until the first IMF $C_{1}(t)=h_{1}(t)$, is obtained.

7. Compute the residual $r_{1}(t)=x(t)-C_{1}(t)$.

8. The residual is treated as the new data and the steps 1 to 7 are repeated until all the IMFs are obtained.

At the end of the algorithm, the EMD decomposition of $x(t)$ is given by:

$$
x(t)=\sum_{j=1}^{N} C_{j}(t)+r_{N}(t)
$$

where $N$ is the number of IMFs, $C_{j}(t)$ is the $j^{t h} \mathrm{IMF}$ and $r_{N}(t)$ is the residue.

\section{PROPOSED METHOD}

\section{A. Conventional EMD-ACWA Approach}

The conventional EMD-ACWA denoising is based on filtering the IMFs resulting from EMD decomposition by the ACWA [1]:

$$
\tilde{C}_{j}(t)= \begin{cases}F_{\text {mean }}+K_{j}\left(C_{j}(t)-F_{\text {mean }}\right), & \text { if } F_{\text {var }} \geq \sigma_{j}^{2} \\ F_{\text {mean }}, & \text { otherwise }\end{cases}
$$

with:

$$
K_{j}=1-\frac{\sigma_{j}^{2}}{F_{\text {var }}}
$$

where $F_{\text {mean }}$ and $F_{\text {var }}$ are the average and the variance of the noisy IMFs computed over an $(L)$ sized window respectively, and $\sigma_{j}^{2}$ is the variance of the noise contained in the $j^{\text {th }}$ noisy
IMF. The absolute median deviation of IMFs is used to estimate the noise variance in this method as follows [16]:

$$
\sigma_{j}^{2}=\left(\frac{\text { meadian }\left(\mid C_{j}(t)-\text { median }\left(C_{j}(t)\right) \mid\right)}{0.6745}\right)^{2}
$$

The denoised signal is the sum of all filtered IMFs.

\section{B. Proposed EMD-ACWA Denoising}

An improved EMD-ACWA denoising scheme is proposed by estimating the noise energies of all IMFs using an energy model, called noise-only model, introduced in [24] and which gives a better estimate of the noise contained in each IMF. Based on EMD studies, it turns out that EMD acts on fractional Gaussian noise as a dyadic filter bank. The noise energy contained in the $j^{\text {th }}$ IMF can be parameterized by a function of the first IMF energy as [24]:

$$
\hat{E}_{j}=\frac{\hat{E}_{1}}{\beta_{H}}\left(\rho_{H}\right)^{-j}, j=2,3 \ldots, N
$$

where $\hat{E}_{1}$ is the noise energy of the first IMF estimated by (4), $\beta_{H}$ and $\rho_{H}$ are experimentally estimated for white Gaussian noise $(H=0.5)$ as $\beta_{H}=0.719$, and $\rho_{H}=2.01$.

On the basis of this model, a new formulation of the conventional method is developed to improve its denoising efficiency. The proposed EMD-ACWA denoising scheme is summarized in the following steps:

1. Perform an EMD decomposition of the noisy signal $x(t)$.

2. Calculate the noise energy contained in the first IMF using (4).

3. Compute the noise energies contained in the rest of IMFs using the noise-only model, as in (5).

4. Filter all IMFs by the ACWA filter over a window of size $(L)$ based on noise energy estimated in each IMF as follows :

$$
\tilde{C} n_{j}(t)= \begin{cases}F_{\text {mean }}+K n_{j}\left(C_{j}(t)-F_{\text {mean }}\right), \text { if } F_{\text {var }} \geq \hat{E}_{j} \\ F_{\text {mean }}, & \text { otherwise }\end{cases}
$$

with:

$$
K n_{j}=1-\frac{\hat{E}_{j}}{F_{v a r}}
$$

5. Reconstruct the denoised signal :

$$
\tilde{x}(t)=\sum_{j=1}^{N} \tilde{C} n_{j}(t)+r_{N}(t)
$$

Figure 1 shows a comparison between the estimated noise energies of IMFs calculated by the median method using (4), and the noise-only model using (5). The signal is contaminated 
by white Gaussian noise with an input Signal to Noise Ratio (SNR) of 4dB. From this Figure, we can see that the noise-only model follows the noise level much better than the median estimation. The median method diverges significantly from the actual energy of noise in the last IMFs (from 6 to 10) that enclose high energy concentration. In addition of a piecewiseregular signal, two other types of test signals and their noisy versions shown in Figure 2 have been used in this section to study the proposed EMD-ACWA denoising approach. Figure 2(a) is a speech signal from Corpora database [22] and Figure 2(b) is an ECG Record of 2048 length chosen from the MITBIH database [23].They are corrupted by white Gaussian noise corresponding to $4 \mathrm{~dB}$ of input SNR. Figure 3 shows the results, together with the achieved SNRs, when the proposed EMDACWA denoising are applied on the noisy signals shown in Figure 1(a), Figure 2(c) and Figure 2(d).

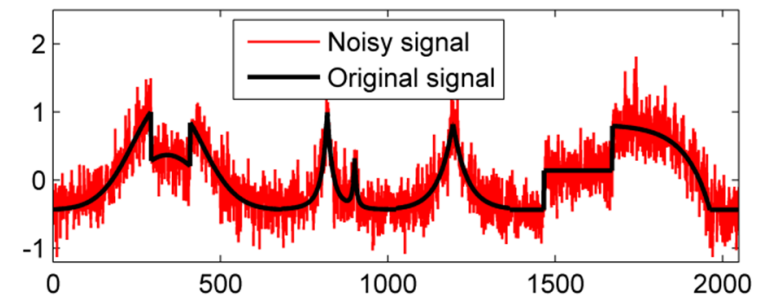

(a)

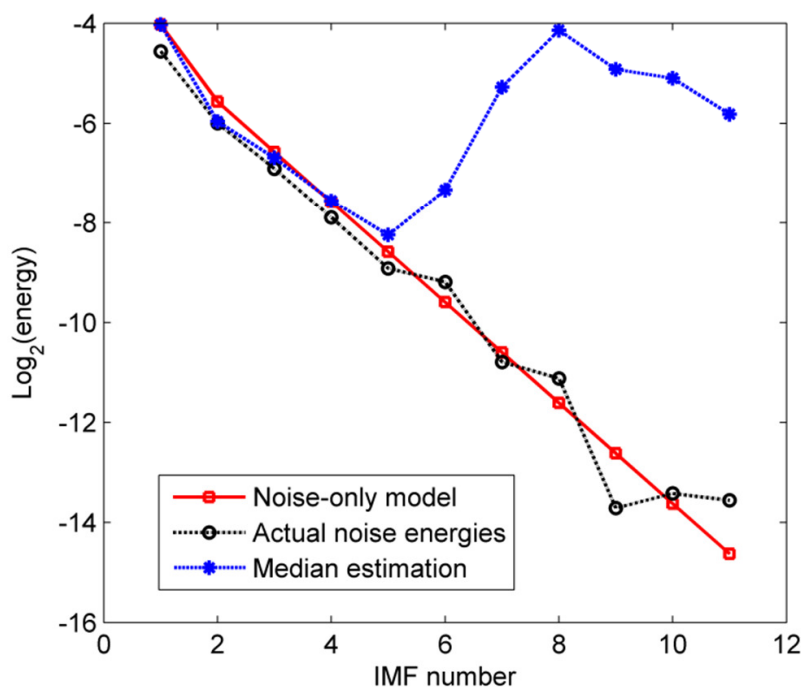

(b)

Fig. 1. (a) A piecewise regular signal and its corresponding noisy signal with input SNR of $4 \mathrm{~dB}$. (b) Comparison of noise estimation obtained by median method and noise-only model

In this example, the size $(L)$ of the ACWA filter window for the proposed EMD-ACWA approach is fixed to 161, 201 and 41 for the piecewise-regular, speech, and ECG signals respectively. The black line is the clean signal, and the red one is the denoised signal. As it's shown, the denoised version of noisy signals tracks the clean one closely, producing significant SNR improvements where the output SNRs have increased to $15.49 \mathrm{~dB}, 12.18 \mathrm{~dB}$ and $14.60 \mathrm{~dB}$ in the cases of the piecewiseregular signal, speech signal and ECG signal respectively.
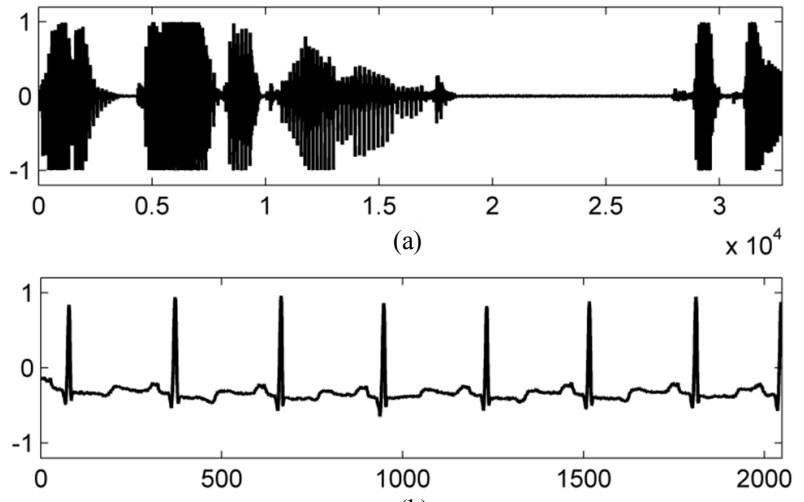

(b)

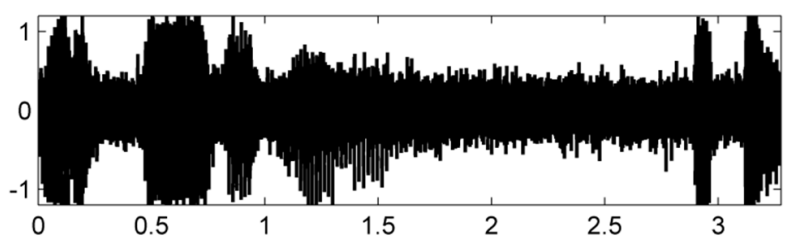

(c)

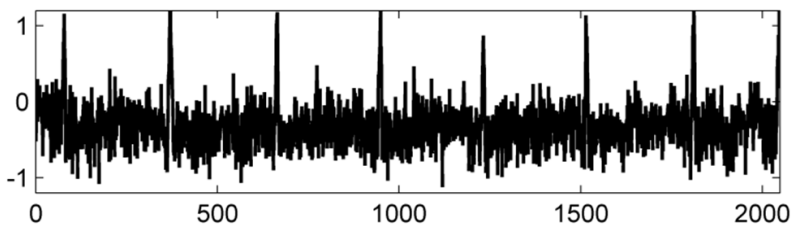

(d)

Fig. 2. (a), (b) Clean versions of a speech signal and ECG record 100. (c), (d) Their noisy versions (input $\mathrm{SNR}=4 \mathrm{~dB}$ )

Figure 4 illustrates the results of denoising when the proposed EMD-ACWA, conventional EMD-ACWA, EMD-IT and wavelet methods are applied to the noisy piecewise regular signal of Figure 1(a). The Daubechies 8 wavelet $(\mathrm{db} 8)$ with a hard threshold as a tool of comparison was used because it gives good results compared to the other wavelets. The black line is the clean signal and the red one is the denoised signal. Clearly, the proposed approach performs better than the other EMD-based methods and the wavelet one. The proposed scheme provides an improvement of about $2.85 \mathrm{~dB}$ compared to the conventional EMD-ACWA.

\section{RESULTS AND DISCUSSION}

In order to study further the efficiency of the proposed method, tests were applied on different simulation and real signals. Besides the piecewise regular signal, "Doppler", "Blocks" and "Bumps" signals are considered in this section (Figure 5). They were generated by the MATLAB function wnoise(fun, $\mathrm{n}$ ) which returns $x(t)$ values of the test signal "fun" (eg. fun=1 for Blocks signal) evaluated at $2^{\mathrm{n}}$ linearly spaced points. Experiments have also been conducted on thirty real speech signals from Corpora Database [22] and thirty real ECG records from the MIT-BIH Arrhythmia Database [23]. ECG and simulation signals are sampled with a sampling frequency that results in 2048 samples. The speech signals are sampled at $16 \mathrm{kHz}$ with $16-$ bit resolution. 


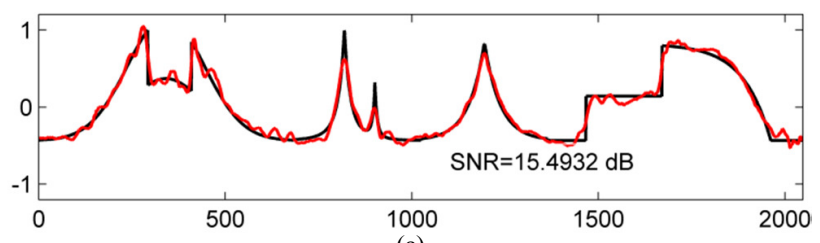

(a)
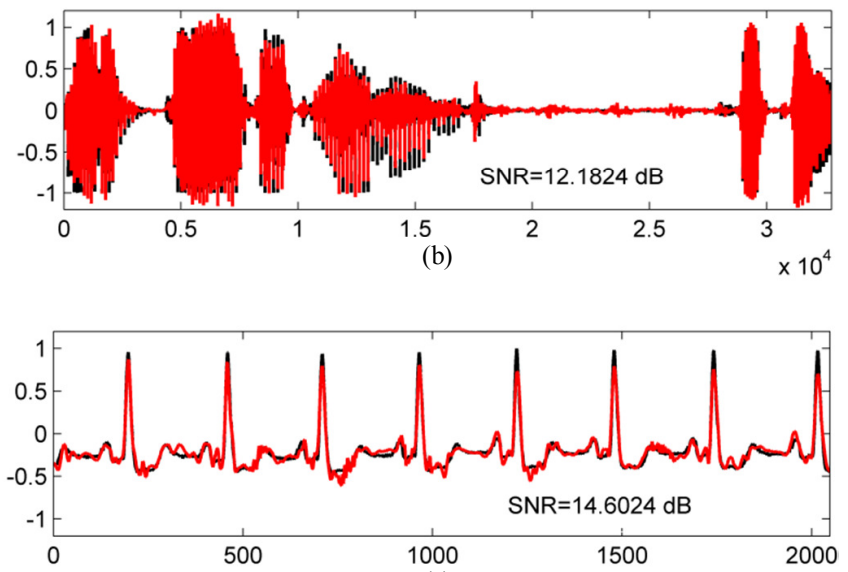

(c)

Fig. 3. Results of the proposed EMD-ACWA denoising. (a) Piecewiseregular, (b) speech signal, (c) ECG signal

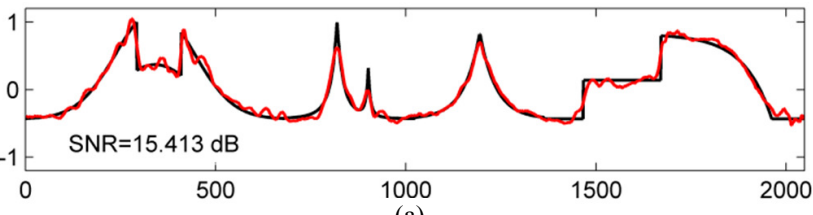

(a)

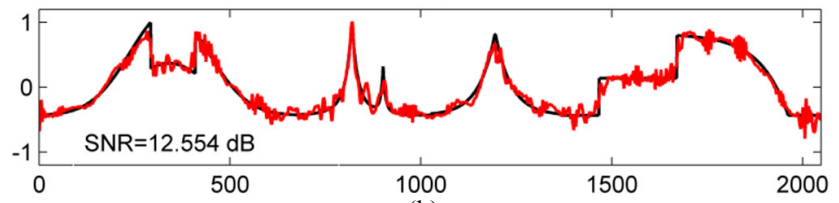

(b)

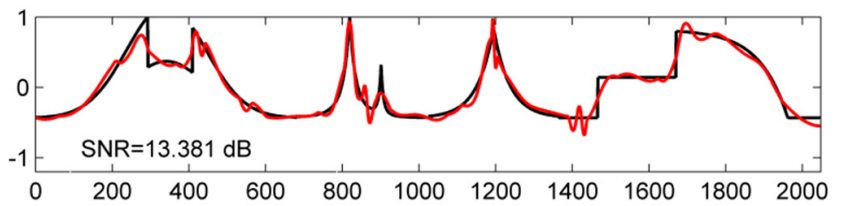

(c)

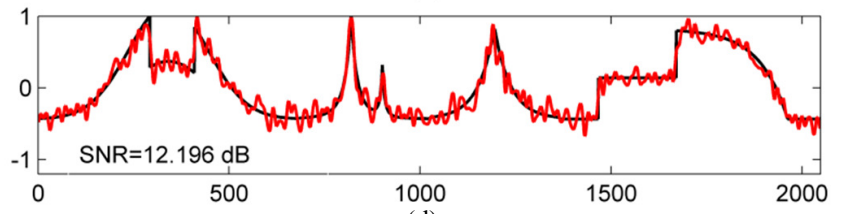

(d)

Fig. 4. Results of the piecewise regular signal denoising. (a) Proposed, (b) conventional EMD-ACWA, (c) EMD-IT, (d) Daubechies 8 wavelet

Figure 6 shows the variation of the output SNR versus the size $(L)$ of the ACWA filter window for different values of input SNR, when the proposed method is applied on the simulation and real signals. The best choice of the window size for simulation signals is $L=161$. In the case of real signals, the best choice of parameter $L$ is 201 for speech signals and 41 for ECG signals. As an evaluation criterion of different methods, we use the SNR after denoising in case of simulation and ECG signals. In case of speech signals, the evaluation is based on SNR and the weighted-slope spectral distance (WSS). All the results presented in this section for the real signals correspond to the average of the ensemble of the results obtained by the different 30 test signals. Noisy versions of the different signals are generated by adding white Gaussian noise to the clean signals with different input SNRs varying from -10 to $8 \mathrm{~dB}$. The best parameters of the size of the ACWA filter window are taken into account when applying the conventional EMDACWA denoising and the proposed method on the different noisy signals.

The performance evaluation of the proposed EMD-ACWA, conventional EMD-ACWA, EMD-IT and wavelet methods when applied on simulated signals is shown in Figure 7. The curves correspond to the output SNR versus the input SNR of different signals: Piecewise regular, Doppler signal, Blocks signal and Bumps signal. The reported results show the effectiveness of the proposed EMD-ACWA method in denoising as it gives a higher enhancement in SNR compared to the other three methods in all cases, especially for low values of input SNR. For relatively high values of input SNR $(>6 \mathrm{~dB})$, the conventional EMD-ACWA method gives the worst denoising results. When applied to the simulated signals, the proposed approach leads to gains between 1 to $4 \mathrm{~dB}$ compared to the conventional EMD-ACWA method, 0.55 to $1.84 \mathrm{~dB}$ compared to EMD-IT one and 1.45 to $7.2 \mathrm{~dB}$ compared to the wavelet one. From these results, we can say then that the proposed denoising method gives the best results.

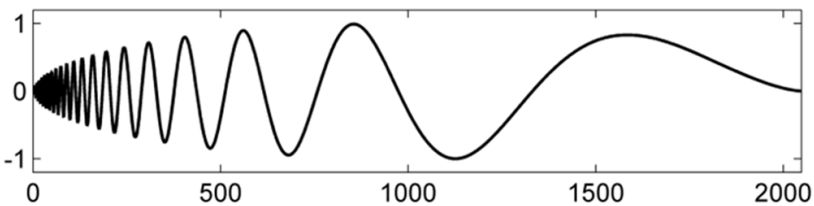

(a)

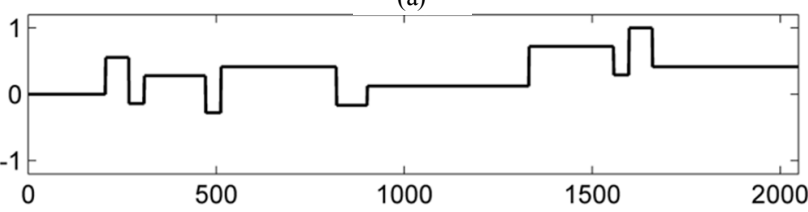

(b)

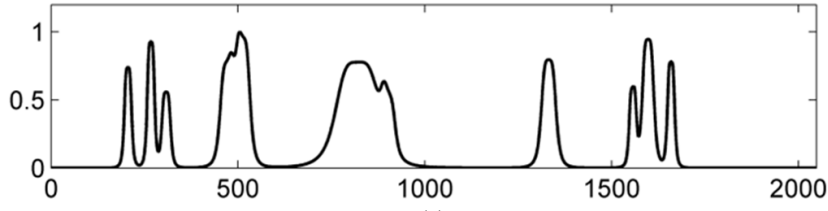

(c)

Fig. 5. Test signals. (a) Doppler, (b) Blocks and (c) Bumps

Figure 8 depicts the denoising results of the proposed method and the other methods considered for comparison when applied on speech and ECG real signals. 

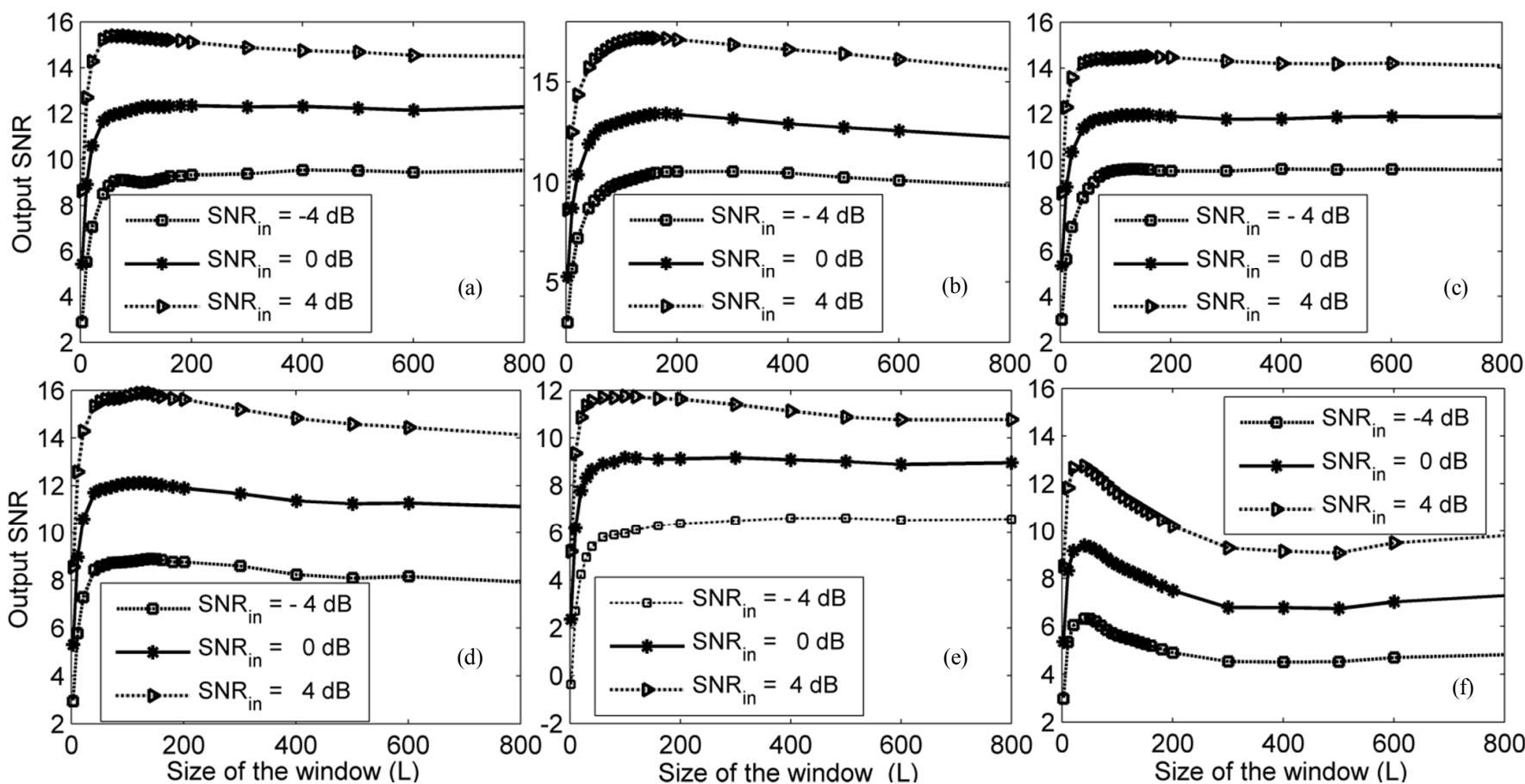

Fig. 6. Effect of the size $(L)$ of the ACWA filter window on the performance of the proposed method when it is applied on simulation and real signals. (a) Piecewise regular, (b) Doppler signal, (c) Blocks signal, (d) Bumps signal, (e) Speech signals, (f) ECG signals
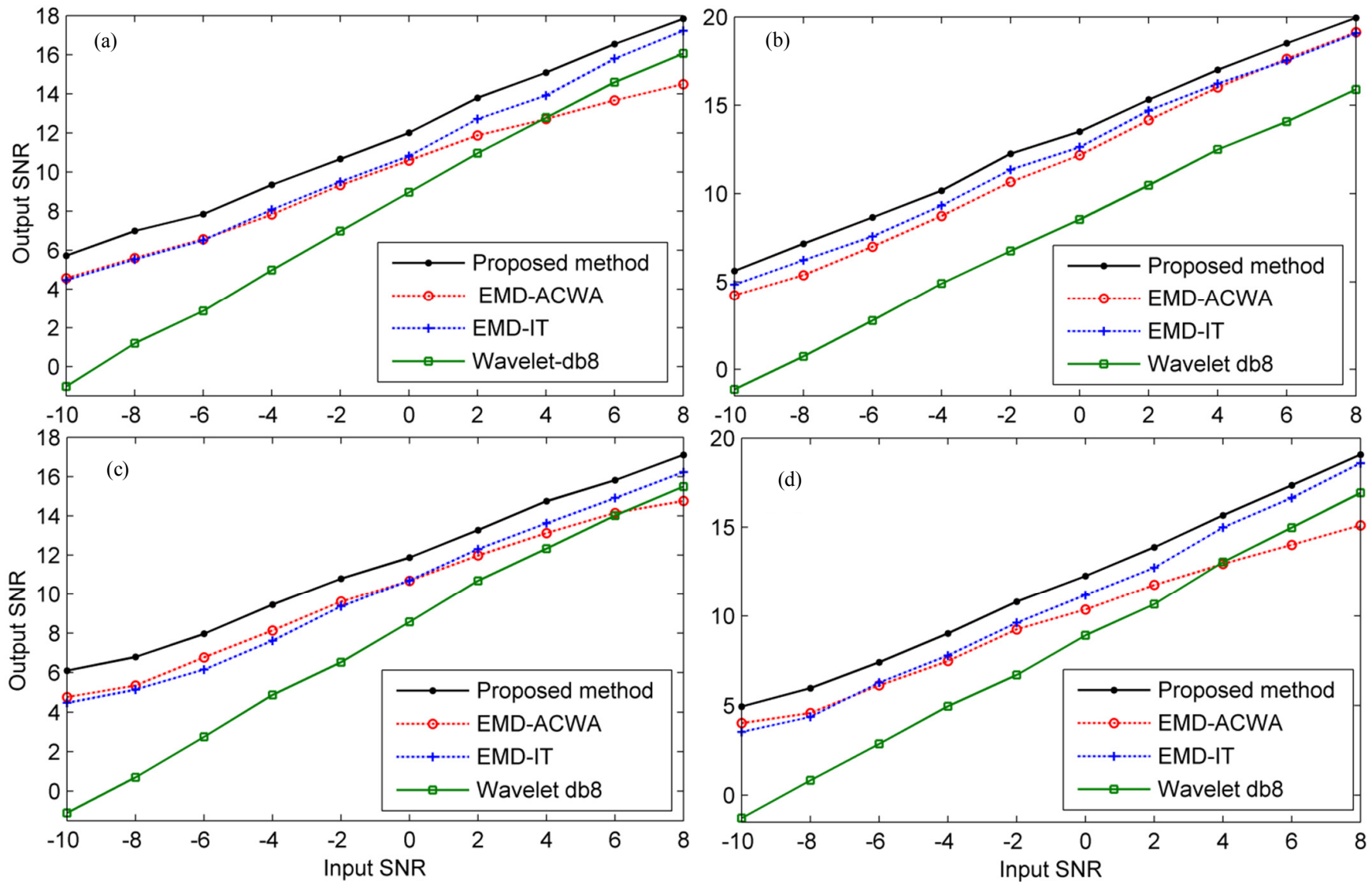

Fig. 7. Performance evaluation of the denoising methods applied on simulated signals. (a) Piecewise regular, (b) Doppler, (c) Blocks, (d) Bumps 
With respect to the real speech signals (Figure 8(a)), the proposed method outperforms the others for almost all SNR levels, especially in range of -6 to $6 \mathrm{~dB}$. The proposed approach offers improvements in output SNR up to $2.85 \mathrm{~dB}$ when compared to the conventional one, $2.45 \mathrm{~dB}$ compared to EMD-IT, and $1.07 \mathrm{~dB}$ when compared to the wavelet method. In regards with the real ECG signals (Figure 8(b)), the conventional EMD-ACWA method gives a very low performance in all cases of input SNR. In contrast, the proposed approach exhibits a significant performance improvement compared to other methods and especially the conventional one. It leads to improvements in output SNR up to $6.95 \mathrm{~dB}$ compared to the conventional EMD-ACWA, $2 \mathrm{~dB}$ compared to the EMD-IT, and $1.86 \mathrm{~dB}$ compared to the wavelet method.
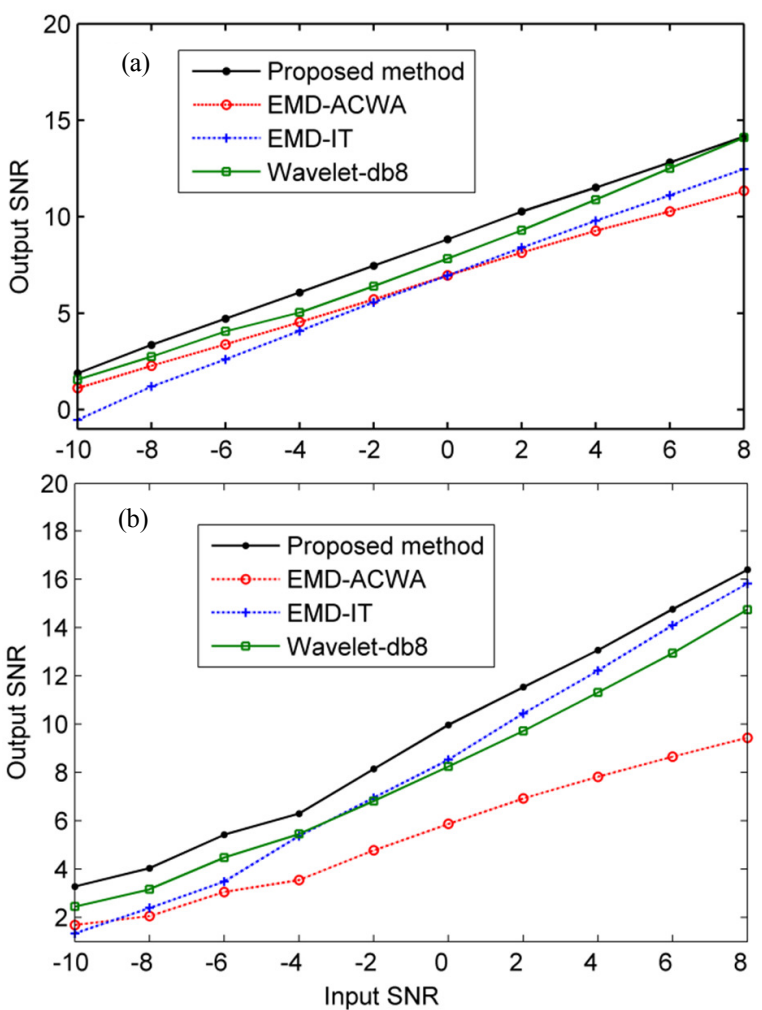

Fig. 8. Performance evaluation of the denoising methods applied on real data. (a) Speech signals, (b) ECG signals

TABLE I. COMPPARISON OF THE WSS DISTANCE

\begin{tabular}{|c|c|c|c|c|}
\hline Input SNR & Proposed & EMD-ACWA & EMD-IT & Wavelet \\
\hline-10 & 57.737 & 63.953 & 141.346 & 202.353 \\
\hline-6 & 52.459 & 58.484 & 107.869 & 166.487 \\
\hline-2 & 45.064 & 53.532 & 75.014 & 115.603 \\
\hline 0 & 41.311 & 50.806 & 65.267 & 92.924 \\
\hline 2 & 38.745 & 48.684 & 58.019 & 84.236 \\
\hline 6 & 32.323 & 43.822 & 41.319 & 59.214 \\
\hline 10 & 26.123 & 37.584 & 33.200 & 39.720 \\
\hline
\end{tabular}

Another measure that characterizes the performance of speech denoising methods is the Weighted-Slope Spectral (WSS) distance [25], which is shown in Table I for different values of input SNR. The proposed EMD-ACWA approach gives the lowest WSS distance which indicates a less distortion degree for all SNR levels. Although the wavelet method gives good results of SNR, it gives inferior quality results of WSS.

\section{CONCLUSION}

In this paper, the ACWA filter was combined with the noise-only model obtained by means of EMD decomposition to improve the denoising performance of the conventional EMDACWA method. The proposed method shows important enhancement in denoising when using the noise only model along with the ACWA filter and choosing the appropriate size of the filter window. To illustrate the effectiveness of the proposed approach, numerous simulations were carried out with different input SNRs, ECG and speech signals. This improved approach exhibits a superior performance compared with EMD-IT, conventional EMD-ACWA, and wavelet methods for all test signals whatever the value of the input SNR. The results were confirmed by the WSS measure in the case of speech signals.

\section{REFERENCES}

[1] K. Khaldi, M. T. H. Alouane, A. O. Boudraa, "Speech denoising by adaptive weighted average filtering in the EMD framework", 2nd International Conference on Signals, Circuits and Systems, Monastir, Tunisia, November 7-9, 2008

[2] M. Rakshit, S. Das, "An efficient ECG denoising methodology using empirical mode decomposition and adaptive switching mean filter", Biomedical Signal Processing and Control, Vol. 40, pp. 140-148, 2018

[3] M. V. Sarode, P. R. Deshmukh, "Image sequence denoising with motion estimation in color image sequences", Engineering, Technology \& Applied Science Research, Vol. 1, No. 6, pp. 139-143, 2011

[4] J. G. Proakis, D. G. Manolakis, Digital signal processing: Principles, algorithms, and applications, Prentice-Hall, 1996

[5] D. L. Donoho, "De-noising by soft-thresholding", IEEE Transactions on Information Theory, Vol. 41, No. 3, pp. 613-627, 1995

[6] T. T. Cai, B. W. Silverman, "Incorporating information on neighbouring coefficients into wavelet estimation", Sankhya: The Indian Journal of Statistics, Vol. 63, No. 2, pp. 127-148, 2001

[7] T. F. Sanam, C. Shahnaz, "Noisy speech enhancement based on an adaptive threshold and a modified hard thresholding function in wavelet packet domain”, Digital Signal Processing, Vol. 23, No. 3, pp. 941-951, 2013

[8] A. Mnassri, M. Bennasr, C. Adnane, "A robust feature extraction method for real-time speech recognition system on a raspberry Pi 3 board”, Engineering, Technology \& Applied Science Research, Vol. 9, No. 2, pp. 4066-4070, 2019

[9] N. E. Huang, Z. Shen, S. R. Long, M. C. Wu, H. H. Shin, Q. Zheng, N. C. Yen, C. C. Tung, H. H. Liu, "The empirical mode decomposition and the Hilbert spectrum for nonlinear and non-stationary time series analysis", Proceedings of the Royal Society of London A, Vol. 454, No. 971, pp. 903-995, 1998

[10] Z. Wu, N. E. Huang, "A study of the characteristics of white noise using the empirical mode decomposition method", Proceedings of the Royal Society of London A, Vol. 460, No. 2046, pp. 1597-1611, 2004

[11] P. Flandrin, G. Rilling, P. Goncalves, "Empirical mode decomposition as filter bank", IEEE Signal Processing Letters, Vol. 11, No. 2, pp. 112 114,2004

[12] G. Rilling, P. Flandrin, P. Goncalves, "Empirical mode decomposition, fractional Gaussian noise and Hurst exponent estimation", IEEE International Conference on Acoustics, Speech, and Signal Processing, Philadelphia, USA, March 23-23, 2005 
[13] B. G. Jeong, B. C. Kim, Y. H. Moon, I. K. Eom, "Simplified noise model parameter estimation for signal-dependent noise", Signal Processing, Vol. 96, No. 2, pp. 266-273, 2014

[14] S. Kumar, D. Panigrahy, P. K. Sahu, "Denoising of electrocardiogram (ECG) signal by using empirical mode decomposition (EMD) with nonlocal mean (NLM) technique", Biocybernetics and Biomedical Engineering, Vol. 38, No. 2, pp. 297-312, 2018

[15] S. Poovarasan, E. Chandra, "Speech enhancement using sliding window empirical mode decomposition and hurst-based technique", Archives of Acoustics, Vol. 44, No. 3, pp. 429-437, 2019

[16] A. O. Boudraa, J. C. Cexus, Z. Saidi, "EMD-based signal noise reduction", International Journal of Signal Processing, Vol. 1, No. 1, pp. 33-37, 2004

[17] A. O. Boudraa, J. C. Cexus, "Denoising via empirical mode decomposition", IEEE International Symposium on Control Communications and Signal Processing, Marrakech, Morocco, March, 2006

[18] Y. Kopsinis, S. Mclanglin, "Development of EMD-based denoising methods inspired by wavelet thresholding", IEEE Transactions on Signal Processing, Vol. 57, No. 4, pp. 1351-1362, 2009

[19] K. Khaldi, M. T. H. Alouane, A. O. Boudraa, "Voiced speech enhancement based on adaptive filtering of selected intrinsic mode functions", Advances in Adaptive Data Analysis, Vol. 2, No. 1, pp. $65-$ 80,2010

[20] J. S. Lee, "Digital image enhancement and noise filtering by use of local statistics", IEEE Transactions on Pattern Analysis and Machine Intelligence, Vol. PAMI-2, No. 2, pp. 165-168, 1980

[21] S. J. Ko, Y. H. Lee, "Center weighted median filters and their applications to image enhancement", IEEE Transactions on Circuits and Systems, Vol. 38, No. 9, pp. 984-993, 1991

[22] http://www.repository.voxforge1.org/downloads/SpeehCorpus/Trunk/Au dio/Main/16kHz_16bit

[23] http: //www.physionet.org/physiobank/database/nstdb

[24] P. Flandrin, G. Rilling, P. Goncalves, "EMD equivalent filter banks, from interpretation to applications", in: Hilbert-Huang Transform and its Applications, World Scientific, pp. 57-74, 2005

[25] D. Klatt, "Prediction of perceived phonetic distance from critical-band spectra: A first step", IEEE International Conference on Acoustics, Speech, and Signal Processing, Paris, France, May 3-5, 1982 